\title{
Variations of Pulsatile Growth Hormone Release in Healthy Short Prepubertal Boys ${ }^{1}$
}

\author{
JAMES R. KERRIGAN, PAUL M. MARTHA, JR., ROBERT M. BLIZZARD, \\ C. MICHELE CHRISTIE, AND ALAN D. ROGOL \\ Departments of Pediatrics [J.R.K., P.M.M., C.M.C., R.M.B.,A.D.R.] and Pharmacology [A.D.R.], University of \\ Virginia Health Sciences Center, Charlottesville, Virginia 22908
}

\begin{abstract}
Overnight growth hormone (GH) concentrations obtained by frequent venous sampling of 20 healthy, short prepubertal boys were evaluated using the objective pulse detection algorithm, CLUSTER. The resulting pulsatile characteristics were compared with those of 11 healthy prepubertal boys of normal stature and with those of nine prepubertal children with documented GH deficiency. Although no significant differences of pulsatile GH release were found between the normal and short subjects, a subset of the short prepubertal boys with significantly delayed skeletal ages had subnormal sum of GH pulse areas and sum of GH pulse amplitudes. The finding of a significant correlation in all subjects between growth velocity and the sum of $\mathrm{GH}$ pulse amplitudes is important, as the results are compatible with the hypothesis that alterations of amplitude-modulated GH release underlie the pathophysiology of suboptimal growth in some short prepubertal children. (Pediatr Res 28: 11-14, 1990)
\end{abstract}

\section{Abbreviations}

GH, growth hormone

DBA, delayed bone age

GHD, growth hormone deficient

$\mathrm{GV}$, growth velocity

IRMA, immunoradiometric assay

SDS, standard deviation score

The adequacy of the hypothalamic-pituitary-GH axis in children has been traditionally assessed using pharmacologic or physiologic stimulation in conjunction with clinical evaluation (1). However, with the advent of repetitive and continuous blood sampling techniques, the evaluation of spontaneous $G H$ release over an extended period of time became possible.

Some investigators have found differences in spontaneous circulating GH levels in some short children when compared with values obtained from children of normal stature (2-5). However, these studies may have been biased by either a failure to properly control for stage of pubertal maturation (3-5) or the inability to precisely characterize the pulsatile pattern of $\mathrm{GH}$ release $(2-5)$. Therefore, we investigated possible abnormalities of spontaneous GH release in healthy short prepubertal boys by

Received August 29, 1989; accepted February 26, 1990.

Correspondence and reprint requests: James R. Kerrigan, M.D. Division of Endocrinology, Department of Pediatrics, MR-4/Room 3037, University of Virginia Health Sciences Center, Charlottesville. VA 22908.

Supponed in part by Diabetes and Hormone Action Training Grant DK-07320 (J.R.K.. P.M.M.), Clinical Investigator Award HD-00868 (P.M.M.), and General Clinical Research Center Grant RR_00847.

Presented in part at the Joint Meeting of the American Pediatric Society and the Society for Pediatric Research, Washington, DC, May 4, 1989. using a closely matched group of healthy prepubertal boys of normal stature and using an objective, statistically-based pulse detection algorithm. A group of GHD children also was studied to provide a more comprehensive spectrum of possible GH pulse abnormalities in prepubertal children with normal and short stature.

\section{MATERIALS AND METHODS}

Forty prepubertal children participated in our study. None had been previously treated with growth-promoting agents. They were separated into the following three groups: normal stature, short stature, and GHD.

Group 1 consisted of 11 healthy boys whose ht and wt were between the 5 th and 95 th percentiles for chronologic age. Skeletal maturities were within two SD for chronologic age.

Group 2 included 20 healthy boys whose ht were less than or equal to the 3rd percentile. Skeletal ages were either normal or delayed for chronologic age, and serum $\mathrm{GH}$ concentrations were $>10 \mu \mathrm{g} / \mathrm{L}$ after provocative testing. None had evidence of systemic illness, intrauterine growth retardation, dysmorphic features, or previous CNS irradiation.

Group 3 consisted of nine GHD children; four males, four females, and a phenotypic female with a 46 XY karyotype. All had ht less than the 3rd percentile for chronologic age, and skeletal maturities were markedly delayed. Maximum $\mathrm{GH}$ concentrations after two provocative stimuli were $\leq 6.0 \mu \mathrm{g} / \mathrm{L}$. Growth velocities were $\leq 4.6 \mathrm{~cm} / \mathrm{y}$.

The Human Investigation Committee at the University of Virginia approved the study. Parents or legal guardians signed consent forms before the study, which was performed in the General Clinical Research Center at the University of Virginia Health Sciences Center. Clonidine, arginine, insulin, levodopa, and/or exercise were used for provocative testing. Stimulation tests were done either more than $24 \mathrm{~h}$ before or immediately after the overnight sampling sessions. Spontaneous GH concentration profiles were assessed beginning on the day of admission by determining the $\mathrm{GH}$ concentrations in serum samples obtained every $20 \mathrm{~min}$ overnight between 2000 and $0800 \mathrm{~h}$ via an i.v. heparin lock needle. Pubertal status was assessed by the method of Marshall and Tanner (6). Skeletal maturity was determined by evaluating a radiograph of the left hand according to the method of Tanner et al. (7). A blood sample for determination of testosterone concentration was obtained at $0600 \mathrm{~h}$.

GH concentrations from the provocative tests were assayed using either the Nichols Institute hGH IRMA with the supplied horse serum matrix (San Juan Capistrano, CA) or the Hybritech two-site IRMA (Hybritech, Inc., San Diego, CA). The lower limit of sensitivity of the Nichols GH assay was $0.5 \mu \mathrm{g} / \mathrm{L}$ with intraand interassay coefficients of variation of $\leq 8.0$ and $\leq 10.0 \%$, respectively. The lower limit of sensitivity of the Hybritech $\mathrm{GH}$ assay was $0.2 \mu \mathrm{g} / \mathrm{L}$ with intra- and interassay coefficients of variation of $\leq 3.8$ and $\leq 5.2 \%$, respectively. $\mathrm{GH}$ determinations 
for the repetitive samplings were performed in duplicate using a GH-IRMA kit supplied by the Nichols Institute. All samples from an individual subject were measured in a single assay run. All samples $\leq 0.5 \mu \mathrm{g} / \mathrm{L}$ were assigned the value of $0.5 \mu \mathrm{g} / \mathrm{L}$ when calculating the mean $\mathrm{GH}$ concentration. Serum testosterone concentrations were determined using a RIA kit obtained from Diagnostic Products Corporation, Los Angeles, CA. IGF-I concentrations were determined using a double antibody RIA kit (Nichols Institute).

The pulsatile characteristics of $\mathrm{GH}$ concentrations were evaluated using the CLUSTER pulse detection algorithm (8) as previously described (9). This program enables one to detect and characterize the significant increases (pulses) that are followed by significant decreases within a given data set. To minimize the false positive rate of peak detection, a one-point by one-point cluster size and a $t$ statistic of 2.32 to evaluate both significant increases and decreases within the data sets were used. Differences among group characteristics were evaluated using analysis of variance with Duncan's multiple range test. Specifically, comparisons were made between four groups that consisted of the normal, GHD, short boys with significant delays of their skeletal maturities ( $n=7$ ), and those short boys with normal skeletal ages ( $n=13$ ). Correlations were assessed by using univariate linear regression analysis. Data management and analysis were performed using the University of Virginia General Clinical Research Center CLINFO facility. Results were reported as mean \pm SEM. Statistical significance was accepted at $p<0.05$.

\section{RESULTS}

The clinical characteristics of the three study groups, as well as those of a subset of seven short boys with significantly DBA (less than the 3 rd percentile or greater than a two-y delay), are shown in Table 1 . Bone age delay was calculated as bone age minus chronologic age in years. Ht and GV, both of which are represented as SDS, were determined using normal values for North American children (10). Ht-SDS and GV-SDS determinations were based on chronologic and skeletal ages, respectively.

The groups did not differ in their mean chronologic or bone ages. The average bone age delay for the GHD group and the DBA subset differed significantly $(p<0.05$ ) from that of both the normal and short groups; values for the normal and short groups were indistinguishable. The ht-SDS for the short boys, the DBA subset, and GHD children were indistinguishable; however, the ht-SDS for these groups differed sigificantly $(p=$ 0.0001 ) from that of the normal group. The GV-SDS of the GHD children was significantly $(p<0.01)$ less than that of all other groups. IGF-I concentrations differed $(p<0.01)$ among the normal, short, and GHD groups. The mean IGF-I concentration of the DBA subset was indistinguishable from that of only the short group. Mean serum testosterone levels were un- detectable $(<0.87 \mathrm{nmol} / \mathrm{L}$ ) in 11 normal, 13 short, and five GHD children whose serum was obtained for this purpose. The seven short boys with DBA could be distinguished from the other 13 short boys (without delayed skeletal maturation) based only on their actual bone age delay $(-2.8 \pm 0.2$ versus $-0.4 \pm 0.2 \mathrm{y} ; p=$ 0.0001 ).

The GH pulsatile characteristics for the three groups, as well as that of the subset of seven short boys with significantly DBA are listed in Table 2. As a group, the short boys had $\mathrm{GH}$ pulsatile characteristics that were indistinguishable from those of the normals, whereas those of the GHD group were significantly less than those of both other groups. However, the subset of short boys with significantly DBA had several GH pulsatile characteristics that were distinguishable from those of both normal and GHD groups. The sum of GH pulse areas and the sum of $\mathrm{GH}$ pulse amplitudes distinguished these seven short boys from the normal and GHD groups. The seven short boys with DBA were distinguishable from the other 13 short boys (without DBA) based only on a lesser mean $\mathrm{GH}$ pulse amplitude $(10.7 \pm 2.3$ versus $16.8 \pm 1.7 \mu \mathrm{g} / \mathrm{L}, p=0.04$ ). Characteristic spontaneous $\mathrm{GH}$ secretory profiles for the study groups are shown in Figure 1.

The GH pulsatile characteristics were also studied for the following subsets of short boys: those with a ht-SDS $\leq-3.0$ ( $n$ $=11)$ and those with a GV-SDS $\leq-2.0(n=7)$. There were no significant differences between the pulsatile characteristics of these two subsets of short boys and those of the normal controls.

Using data obtained from the 20 short boys, we found a significant correlation between bone age delay and the sum of GH pulse amplitudes ( $r=0.48, p=0.03$ ) as shown in Figure 2 . The correlation coefficient and $p$ value were not changed apprecrably when the value of $117 \mu \mathrm{g} / \mathrm{L}$ was omitted before regression analysis. No significant correlations between GV-SDS or ht-SDS and any of the GH pulse characteristics for the individual study groups were found. However, when analyzing data combined from all study groups, significant relationships were found between ht and the sum of GH peak areas $(r=0.46, p=0.02)$, between ht-SDS and the sum of GH peak areas $(r=0.38, p=$ 0.02 ), and between GV-SDS and the logarithm of the sum of GH pulse amplitudes $(r=0.45, p<0.01)$.

\section{DISCUSSION}

The observation by several investigators $(11,12)$ that the physiologic pattern of $\mathrm{GH}$ release in children is represented as a continuum, rather than as a bimodal distribution, may provide an explanation for the difficulty encountered in attempting to distinguish spontaneous $\mathrm{GH}$ secretory patterns among groups of short children. Therefore, the GH secretory profiles of the group of short boys that we studied were not distinguishable from those of the normal controls. However, the pulsatile patterns of $\mathrm{GH}$

Table 1. Clinical characteristics of study groups*

\begin{tabular}{|c|c|c|c|c|c|c|}
\hline Study group & $\begin{array}{c}\text { Chronofogic age } \\
\text { (y) }\end{array}$ & $\begin{array}{c}\text { Bone age } \\
(y)\end{array}$ & $\begin{array}{c}\text { Bone age delay } \\
(y)\end{array}$ & $\begin{array}{c}\mathrm{Ht} \\
(\mathrm{SDS})\end{array}$ & GV (SDS) & $\begin{array}{c}\text { IGF-I } \\
(\mathrm{IU} / \mathrm{mL})\end{array}$ \\
\hline $\begin{array}{l}\text { Normal } \\
(n=11)\end{array}$ & $\begin{array}{r}9.0 \pm 0.3 \\
(7.2-10.0)\end{array}$ & $\begin{array}{r}8.2 \pm 0.4 \\
(6.0-10.5)\end{array}$ & $\begin{array}{l}-0.8 \pm 0.2 \\
(-2.1-0.5)\end{array}$ & $\begin{array}{r}0.1 \pm 0.3 \\
(-1.0-1.2)\end{array}$ & $\begin{array}{c}0.6 \pm 0.8 \\
(-2.8-2.5)\end{array}$ & $\begin{array}{c}0.90 \pm 0.13 \\
(0.34-1.77)\end{array}$ \\
\hline $\begin{array}{l}\text { Short } \\
(n=20)\end{array}$ & $\begin{array}{r}9.8 \pm 0.5 \\
(5.6-13.9)\end{array}$ & $\begin{array}{r}8.6 \pm 0.5 \\
(3.5-12.1)\end{array}$ & $\begin{array}{l}-1.2 \pm 0.3 \\
(-3.9-1.5)\end{array}$ & $\begin{array}{l}-2.8 \pm 0.6^{4} \\
(-3.9-1.9)\end{array}$ & $\begin{array}{l}-0.9 \pm 0.4 \\
(-3.6-2.1)\end{array}$ & $\begin{array}{l}0.60 \pm 0.06^{ \pm} \\
(0.22-1.18)\end{array}$ \\
\hline $\begin{array}{l}\text { GHD } \\
(n=9)\end{array}$ & $\begin{array}{l}10.5 \pm 1.3 \\
(4.8-15.3)\end{array}$ & $\begin{array}{r}8.2 \pm 1.4 \\
(2.2-14.3)\end{array}$ & $\begin{array}{l}-2.3 \pm 0.4^{a} \\
(-4.8-1.0)\end{array}$ & $\begin{array}{c}-3.2 \pm 0.4^{a} \\
(-4.5--2.0)\end{array}$ & $\begin{array}{l}-3.8 \pm 0.7^{\mathrm{a}} \\
(-6.8-0.0)\end{array}$ & $\begin{array}{l}0.21 \pm 0.02^{\mathrm{b}} \\
(0.13-0.29)\end{array}$ \\
\hline
\end{tabular}

* Values are mean \pm SEM; numbers in parentheses are ranges. Any value followed by a superscript differs significantly $(p<0.05)$ from all other values in the same column not followed by the same superscript. Refer to Results section for explanation of statistical comparisons.

t Subset of the short group. 